\title{
Maffucci syndrome complicated by three different central nervous system tumors sharing an IDHI R132C mutation: case report
}

\author{
Takahide Nejo, MD, ${ }^{1}$ Shota Tanaka, MD, ${ }^{1}$ Masako Ikemura, MD, PhD, ${ }^{2}$ Masashi Nomura, MD, PhD, ${ }^{1}$ \\ Shunsaku Takayanagi, MD, PhD, ${ }^{1}$ Masahiro Shin, MD, PhD, ${ }^{1}$ Tetsuo Ushiku, MD, PhD, ${ }^{2}$ \\ Junji Shibahara, MD, PhD, ${ }^{2}$ Nobuhito Saito, MD, PhD, ${ }^{1}$ and Akitake Mukasa, MD, PhD ${ }^{1}$ \\ Departments of ${ }^{1}$ Neurosurgery and ${ }^{2}$ Pathology, Graduate School of Medicine, The University of Tokyo, Japan
}

\begin{abstract}
Maffucci syndrome (MS) and Ollier disease (OD) are nonhereditary congenital diseases characterized by multiple enchondromas and/or chondrosarcomas. Recent studies have implicated somatic mosaic mutations of isocitrate dehydrogenase 1 or 2 (IDH1/2) as contributing to the pathogenesis of MS and OD. Occasionally, patients with these disorders may also present with central nervous system (CNS) tumors; however, detailed genetic analyses are limited. In this article, the authors report on a male patient with MS, harboring three CNS tumors that share a common genetic alteration. Over a 9-year period, three separate tumor resections were conducted for sellar, intraparenchymal brainstem, and osseous clival tumors. The histopathological diagnoses were pituitary adenoma, diffuse astrocytoma, and chondrosarcoma, respectively. Sanger sequencing revealed a common IDH1 R132C mutation among all three CNS tumors but not in blood DNA. Administering chemotherapy (nimustine) and subsequent radiation therapy to the brainstem glioma and the residual lesion in the clivus have kept the patient progression free for 18 months. This is the first report demonstrating an IDH1 mutation shared among three different CNS tumors in a single patient with MS. The findings support the hypothesis that in MS and OD, a single common IDH1 mutation triggers tumorigenesis in cells of different origins and locations in a somatic mosaic fashion.
\end{abstract}

https://thejns.org/doi/abs/10.3171/2018.6.JNS18729

KEYWORDS Maffucci syndrome; isocitrate dehydrogenase; diffuse astrocytoma; chondrosarcoma; pituitary adenoma; oncology

I N 2008, Parsons et al. first reported a mutation in the isocitrate dehydrogenase 1 (IDHI) gene in glioblastomas and showed that it was associated with a favorable prognosis. ${ }^{17}$ This mutation, along with a rare mutation in the isocitrate dehydrogenase 2 (IDH2) gene, turned out to be a hallmark of grade II and III gliomas and secondary glioblastomas. ${ }^{11,23,24}$ Subsequent studies revealed that IDHI/2 mutations were also found in several other types of tumors. ${ }^{24}$

Among them, cartilaginous tumors are known to have a high frequency of $I D H 1 / 2$ mutations, comparable to that of gliomas. They involve the rare nonhereditary congenital diseases Maffucci syndrome (MS) and Ollier disease
(OD), which are characterized by enchondromatosis often with asymmetrical distribution.1,19 MS is distinguished from OD by the appearance of soft-tissue vascular lesions, which accompany MS but not OD. Recent largescale genetic analyses have shown that $77 \%-93 \%$ of tumors in these diseases have $I D H 1 / 2$ mutations, implicating IDH somatic mosaic mutations, which occur at the early postzygotic period, as the major factors in the pathogenesis of these diseases. ${ }^{2,16}$

While most tumors in MS and OD affect mesodermal tissues, they are sometimes accompanied by lesions in the neuroendocrine system. Several cases with central nervous system (CNS) tumors have been reported, including

ABBREVIATIONS ATRX $=\alpha$-thalassemia/mental retardation syndrome $x$-linked; CNS $=$ central nervous system; D2HG $=\mathrm{D}$-2-hydroxyglutarate; EMA $=$ epithelial membrane antigen; $M S=$ Maffucci syndrome; $O D=$ Ollier disease.

SUBMITTED March 28, 2018. ACCEPTED June 27, 2018.

INCLUDE WHEN CITING Published online December 21, 2018; DOI: 10.3171/2018.6.JNS18729. 


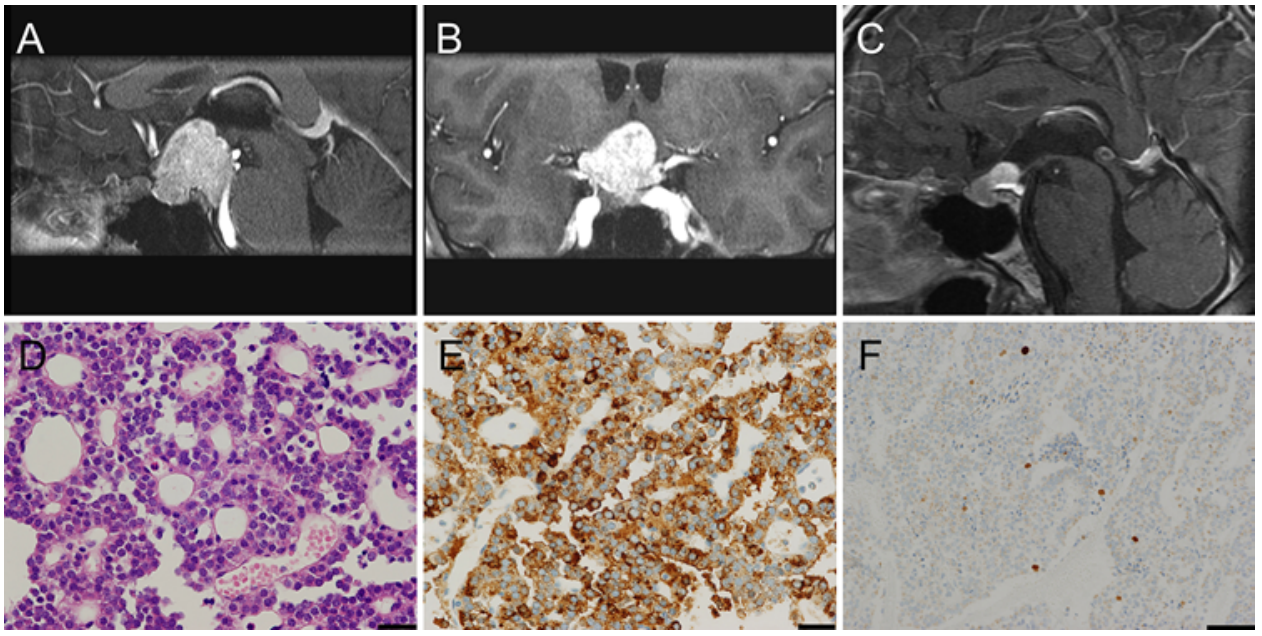

FIG. 1. Tumor 1. Sagittal (A) and coronal (B) T1-weighted MR images with gadolinium demonstrate a homogeneously enhancing lesion arising from the sella turcica and extending posterosuperiorly. A postoperative image (C) reveals a small amount of residual tumor around the stalk, indicating subtotal resection. An $\mathrm{H} \&$ E-stained section (D) shows tumor cells with monomorphous round nuclei in a papillary and cord-like arrangement. Tumor cell staining was strongly positive for the neuroendocrine marker chromogranin A (E). The Ki-67 labeling index was 1\% (F). Bar = $25 \mu \mathrm{m}(\mathrm{D}-\mathrm{E})$ or $100 \mu \mathrm{m}(\mathrm{F})$. Figure is available in color online only.

glioma, pituitary adenoma, olfactory neuroblastoma, spindle cell hemangioendothelioma, and chondrosarcoma. ${ }^{19}$ However, detailed genetic analysis on these CNS tumors in MS and OD is still very limited. ${ }^{8}$

Here, we report a case of MS complicated by three different CNS tumors in which common genetic alterations were noted. We further discuss IDH-related tumorigenesis with a review of the literature.

\section{Case Report}

Tumor 1: Sellar Tumor

A 31-year-old man with diplopia was referred to our hospital. He had been diagnosed with MS at 3 years of age given multiple enchondromas and a subcutaneous hemangioma on his right upper and lower extremities, for which he had undergone orthotopic tumor resections for a total of eight times. Physical examination revealed abducens nerve palsy on the right side; functions of the other cranial nerves were normal. No abnormal findings were seen on serum laboratory testing for pituitary hormones and related peptides. Magnetic resonance imaging showed a 31-mm mass located in the sellar region with homogeneous enhancement after gadolinium administration on T1-weighted imaging (Fig. 1A and B). Subtotal resection of the enhancing lesion was performed via a transsylvian approach given the predominantly suprasellar location of the tumor and the differential diagnoses such as chondrosarcoma or meningioma (Fig. 1C). Thereafter, the patient's symptoms gradually improved.

Histologically, the tumor consisted of small, monomorphous round cells arranged in a papillary and cordlike manner (Fig. 1D). On immunohistochemistry, tumor cells were strongly immunoreactive for synaptophysin and chromogranin A (Fig. 1E); the Ki-67 labeling index was $1 \%$ (Fig. 1F). Based on these findings, a diagnosis of nonfunctioning pituitary adenoma was made.
While a tiny amount of residual tumor was present on postoperative MRI, considering the benign diagnosis, a wait-and-see strategy was chosen.

\section{Tumor 2: Brainstem Tumor}

The patient underwent an annual imaging checkup after his first CNS surgery because an intraparenchymal, T2-hyperintense, nonenhancing lesion in the pons had been pointed out during his first visit (Fig. 2A). After 7 years from the previous CNS surgery, marked longitudinal enlargement of the lesion, as well as formation of another tumor in the petroclival area, was noted (Fig. 2B). The lesion remained nonenhancing with gadolinium administration (not shown). Because the clinical course was indolent and seemed atypical for diffuse midline glioma, a stereotactic biopsy through the right cerebellar hemisphere was performed for diagnostic purposes.

Histologically, the tumor consisted of mildly atypical glial cells with naked nuclei and little cytoplasm where normal structure interposed (Fig. 2C). The density of the atypical cells was relatively low. Mitosis, necrosis, or microvascular proliferation was not observed. The Ki-67 labeling index was less than $3 \%$. Owing to histopathological examination and genetic analysis detailed in Genetic Analysis below (Figs. 2D and E and 4B), we finally made the diagnosis of diffuse astrocytoma, IDH mutant, WHO grade II.

Given the diagnosis, treatment with nimustine alone was chosen, and radiotherapy was postponed at that time because of the possible necessity for subsequent irradiation to the clival lesion. The lesion gradually diminished on T2-weighted MRI after eight cycles of nimustine treatment (Fig. 2F).

\section{Tumor 3: Skull Base Osseous Tumor}

When the patient was 40 years of age, 21 months after the second CNS surgery, his right abducens nerve palsy worsened again. MRI demonstrated that the known dor- 

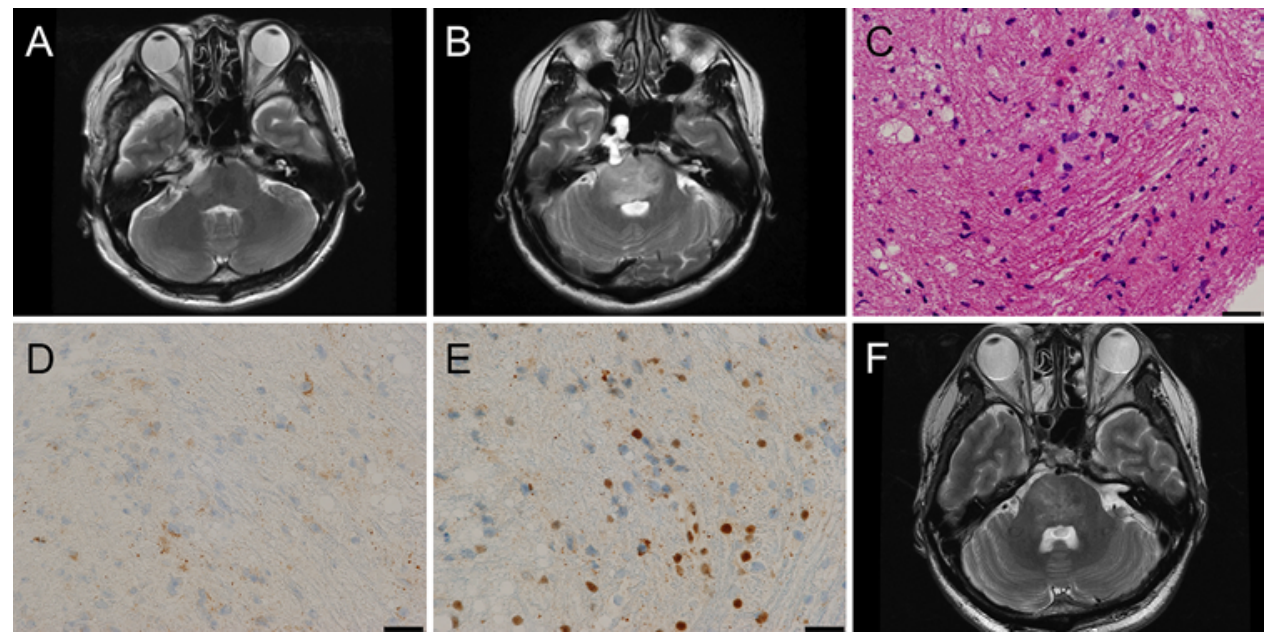

FIG. 2. Tumor 2. Axial T2-weighted MR images taken at the patient's initial visit (A) and 7 years later $(\mathbf{B})$, revealing enlargement of the hyperintense lesion in the pons. An $\mathrm{H}$ \& E-stained section (C) obtained through stereotactic biopsy shows that the tumor consisted of mildly atypical glial cells with naked nuclei and little cytoplasm where normal structure interposed. No obvious high-grade features were seen. On immunohistochemistry, tumor cells stained negative for IDH1 R132H (D). ATRX ( $\alpha$-thalassemia/mental retardation syndrome $x$-linked) expression was lost in tumor cells but retained in the endothelium and other normal cells (E). On MRI performed after eight courses of chemotherapy, shrinkage of the lesion was observed (F). Magnetic resonance images (B and $\mathrm{F}$ ) indicated that another tumor (tumor 3 ) had developed in the dorsal petroclivus on the right side. Bar $=25 \mu \mathrm{m}(\mathrm{C}-\mathrm{E})$. Figure is available in color online only.

sal petroclival osseous tumor had grown and expanded posteriorly (Fig. 3A and B). Endoscopic transnasal tumor resection was performed, ${ }^{9,20}$ and near-total resection of the enhancing lesion was achieved (Fig. 3C).

Histologically, the tumor consisted of atypical cells with circular hyperchromatic nuclei and eosinophilic cytoplasm in a chondromyxoid background (Fig. 3D). Binucleation was rarely seen and mitosis was not observed. On immunohistochemistry, tumor cells stained negative for epithelial membrane antigen (EMA). The Ki-67 labeling index was approximately $1 \%$. Collectively, the diagnosis of chondrosarcoma, WHO grade II was made. Subsequently, the patient received radiation at 54 Gy in 27 fractions to the brainstem glioma and the residual lesion in the clivus. He was stable without any worsening neurological symptoms and remained progression free at 18 months after the last surgery.

\section{Tumor Material}

Tumor samples and paired blood samples were obtained and analyzed according to the institutional guidelines for the study of human tissues. Clinical data were retrieved from electronic medical records. This study was approved by the research ethics committee of The University of Tokyo. The patient provided written informed consent for the genetic analysis. Histological diagnoses were made by senior neuropathologists (M.I., T.U., and J.S.) for all three tumors according to the WHO classification.

\section{Immunohistochemistry}

Sections from formalin-fixed, paraffin-embedded tissue blocks were subjected to $\mathrm{H} \& \mathrm{E}$ staining for histopathological diagnosis and to immunohistochemical analysis, using a Ventana BenchMark XT automated immunostain- er (Roche). We used 3- $\mu$ m-thick sections according to the departmental standard protocol. The following antibodies were applied: synaptophysin (monoclonal, MRQ-40, Roche), chromogranin A (polyclonal, 1:500, DAKO), Ki67 (monoclonal, MIB-1, DAKO), IDH1 R132H (monoclonal, clone H09, 1:20, dianova), histone H3 K27M (polyclonal, 1:200, Merck Millipore), $\alpha$-thalassemia/mental


FIG. 3. Tumor 3. Axial (A) and sagittal (B) T1-weighted MR images with gadolinium reveal a heterogeneously enhancing lesion protruding into the anterior surface of the brainstem. A postoperative axial image (C) indicates near-total resection of the contrast-enhancing lesion. An $\mathrm{H}$ \& E-stained section (D) shows atypical cells with circular hyperchromatic nuclei and eosinophilic cytoplasm in the chondromyxoid background. Binucleation was rarely seen and no mitosis was observed. Bar $=50 \mu \mathrm{m}$ (D). Figure is available in color online only. 


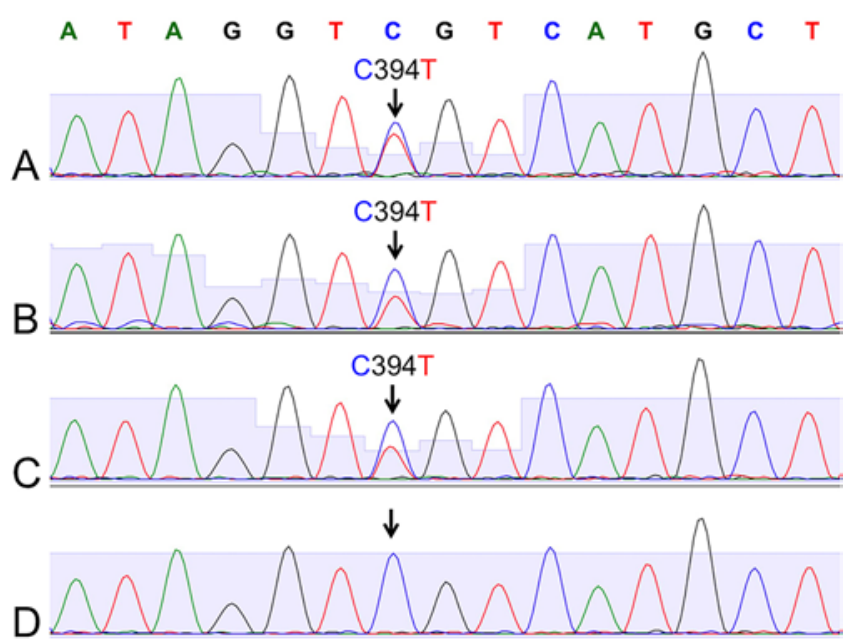

FIG. 4. Sanger sequencing for IDH1 on the three tumors (pituitary adenoma, A; brainstem diffuse astrocytoma, B; skull base chondrosarcoma, C) and normal DNA (D). IDH1 c.394C > T (R132C) alteration was consistently demonstrated on all three tumors. On the other hand, IDH1 codon 132 sequence in the DNA from peripheral blood sample was confirmed to be wild type (D). Figure is available in color online only.

retardation syndrome x-linked (ATRX; polyclonal, 1:500, Sigma-Aldrich), and EMA (monoclonal, E29, Roche).

\section{Genetic Analysis}

The QIAamp DNA Mini Kit (Qiagen) was used to extract genomic DNA from fresh-frozen tumor samples (tumors 1-3) and from a blood sample, according to the manufacturer's protocols. The presence of hotspot mutations in IDH1 (R132), IDH2 (R172), and the telomerase reverse transcriptase (TERT) gene promoter (C228 and C250) was assessed on each sample using Sanger sequencing. An aliquot of genomic DNA was amplified by polymerase chain reaction using previously reported oligo primers. ${ }^{3,15}$ Sequencing was performed by FASMAC Co. Ltd., who used the Applied Biosystems 3730xl or 3130xl DNA analyzer (Thermo Fisher Scientific). Loss of heterozygosity on chromosomes $1 p$ and $19 q$ was examined for the second tumor (tumor 2) using microsatellite analysis, as described previously.22

Sanger sequencing for $I D H I / 2$ demonstrated that the missense mutation IDHI R132C (c.394 C > T) was shared among all three tumors (Fig. 4A-C). No IDH2 mutations were detected. Both $I D H I$ and $I D H 2$ were wild type in blood DNA (Fig. 4D), supporting the hypothesis that the major pathogenesis of MS is IDH somatic mosaic mutation.

Along with IDHI mutation and ATRX loss (Fig. 2E) and negative staining of histone H3 K27M on immunohistochemistry, retention of chromosome $1 p$ and $19 q$ in microsatellite analysis confirmed the diagnosis of diffuse astrocytoma, IDH mutant in tumor 2. By contrast, ATRX expression was retained in the other two tumors (not shown). In these two ATRX-intact tumors, TERT promoter mutation status was also explored and judged as wild type in both samples (Table 1). MGMT promoter methylation status was not examined, even for the brainstem glioma, owing to the lack of sufficient tissue to perform the assessment.

\section{Discussion}

To our knowledge, this is the first report of MS complicated by three different CNS tumors that shared the IDHI R132C mutation. While MS and OD cases can involve multiple CNS tumors, previous reports have lacked evidence of genetic commonality among tumors. ${ }^{18,19}$ The current case may help us to gain new insight into IDH-related tumorigenesis in these diseases.

Compared with glioma and chondrosarcoma, little is known about the role that IDH mutation plays in the pathogenesis of pituitary adenoma. Several investigators have failed to demonstrate IDH mutations in pituitary adenomas using immunohistochemistry and/or genetic analyses, ${ }^{5,6,13}$ suggesting that IDH mutations play a limited role in the pathogenesis of sporadic pituitary adenomas. Hao et al. recently illustrated an MS case accompanied by an intracranial chondrosarcoma and a pituitary adenoma, both of which harbored the common IDH1 R132C mutation. ${ }^{8}$ Prior to the present case, to the best of our knowledge, theirs was the only report on pituitary adenoma in which the $I D H I$ mutation was genetically demonstrated. Additionally, they reviewed twelve previously reported MS cases with pituitary adenoma, but all lacked genetic exploration. However, the clear evidence of $I D H I$ mutation in pituitary adenomas in those two cases, the high incidence of this tumor in MS and OD, and the proposed model that $I D H 1 / 2$ somatic mosaic mutation is the main

TABLE 1. Pathological and molecular characteristics of the three tumors

\begin{tabular}{|c|c|c|c|c|}
\hline Variable & Tumor 1 & Tumor 2 & Tumor 3 & Normal (blood) \\
\hline Histological diagnosis & Pituitary adenoma & Diffuse astrocytoma, IDH mutant, grade II & Chondrosarcoma, grade II & \\
\hline IDH1 R132H (IHC) & NA & Negative & NA & \\
\hline IDH1 (Sanger) & $\mathrm{R} 132 \mathrm{C}$ (c.394C > T) & $\mathrm{R} 132 \mathrm{C}(\mathrm{c} .394 \mathrm{C}>\mathrm{T})$ & $\mathrm{R} 132 \mathrm{C}$ (c.394C > T) & Wild type \\
\hline IDH2 (Sanger) & Wild type & NA & Wild type & Wild type \\
\hline $\operatorname{ATRX}(\mathrm{IHC})$ & Retained & Lost & Retained & \\
\hline 1p19q (microsatellite analysis) & NA & Intact & NA & \\
\hline TERT promoter (Sanger) & Wild type & NA & Wild type & \\
\hline
\end{tabular}

$\mathrm{IHC}=$ immunohistochemistry; $\mathrm{NA}=$ not available. 
cause of these diseases collectively suggest that IDH mutation contributes to the pathogenesis of pituitary adenomas, at least under such particular circumstances.

However, subsequent genetic events of IDH mutation in tumorigenesis in MS and OD remain unclear. In gliomas, mutations in TP53 and ATRX are supposed to be very early events following IDH mutation in tumorigenesis in grade II-III IDH-mutant astrocytomas. ${ }^{21}$ Indeed, ATRX loss on immunohistochemistry was observed in tumor 2 in the current case. This observation is in contrast to ATRX retention in tumors 1 and 3. Considering the mutual exclusivity with $A T R X$ mutation, we then looked for TERT promoter hotspot mutations (C228 and C250) in tumors 1 and 3 but identified both as wild type. Similarly, Moriya et al. described an MS case with enchondromas, cutaneous hemangioma, and anaplastic astrocytoma, in which the IDH2 R172S mutation was shared among those tumor samples, but a TP53 missense mutation was detected exclusively in the astrocytoma. ${ }^{14}$ These findings indicate that genetic events subsequent to IDHI/2 mutation are not shared between gliomas and other types of tumors in MS and OD.

Epigenetic changes may precede genetic ones as events leading to tumorigenesis in IDH-mutant tumors. The IDH1/2 mutant enzymes convert $\alpha$-ketoglutarate $(\alpha K G)$ into D-2-hydroxyglutarate (D2HG), which is considered an oncometabolite and competitively inhibits the enzymatic activity of many $\alpha \mathrm{KG}$-dependent dioxygenases: 1) inhibition of KDM6A and JmjC domain-containing demethylases leads to histone dysregulation; 2) inhibition of TET2 brings genome-wide DNA hypermethylation; and 3) inhibition of prolyl hydroxylase domain (PHD) triggers aberrant angiogenesis through upregulation of hypoxia-inducible factor 1-alpha (HIF-1 $\alpha)^{4,7,12,24}$ Amary et al. reported that among 40 individuals with MS or OD, IDH-mutant chondrosarcomas contained significantly higher levels of D2HG compared to their IDH wild-type counterparts, implicating it as an oncometabolite in these tumors. $^{2}$ At the same time, Pansuriya et al., using Illumina HumanMethylation27 BeadChip analysis, showed that seven of eight IDH-mutant enchondromas in MS and OD cases were comparable to the $\mathrm{CpG}$ island methylator phenotype (CIMP). ${ }^{16}$ They argued that genome-wide hypermethylation and downregulated expression of several genes induced by mutant IDH and elevated 2HG play a crucial role in tumorigenesis in MS and OD. In addition, Hirata et al. argued that IDHI mutation or elevated D2HG was sufficient to develop enchondroma-like lesions by upregulation of hedgehog signaling pathways in their IDHI conditional knock-in mouse model. ${ }^{10}$ Comprehensive genetic and epigenetic analyses on those different tumors of MS and OD will yield important insight into IDH-related tumorigenesis, raising hope for the development of molecular targeted agents in the treatment of MS and OD.

\section{Conclusions}

In summary, for the first time, we have demonstrated a shared IDHI R132C mutation among three different CNS tumors in a single MS case. Our findings support the hypothesis that the single common IDHI mutation triggers tumorigenesis in cells of different origin at different locations in a somatic mosaic fashion in MS and OD. Because no subsequent shared genetic events were identified among the three tumors from our limited analyses, further comprehensive investigation is warranted to uncover IDHassociated tumorigenesis.

\section{Acknowledgments}

We are grateful to the patient for donating the tissue samples for our research, and we acknowledge the excellent technical assistance of Reiko Matsuura and Mayu Omata (Department of Neurosurgery, Graduate School of Medicine, The University of Tokyo). This work was supported by the Project for Cancer Research and Therapeutic Evolution (P-CREATE; A. Mukasa and S. Tanaka) from the Japan Agency for Medical Research and Development (AMED).

\section{References}

1. Amary MF, Bacsi K, Maggiani F, Damato S, Halai D, Berisha F, et al: IDH1 and IDH2 mutations are frequent events in central chondrosarcoma and central and periosteal chondromas but not in other mesenchymal tumours. J Pathol 224:334-343, 2011

2. Amary MF, Damato S, Halai D, Eskandarpour M, Berisha F, Bonar F, et al: Ollier disease and Maffucci syndrome are caused by somatic mosaic mutations of IDH1 and IDH2. Nat Genet 43:1262-1265, 2011

3. Arita H, Narita Y, Fukushima S, Tateishi K, Matsushita Y, Yoshida A, et al: Upregulating mutations in the TERT promoter commonly occur in adult malignant gliomas and are strongly associated with total $1 \mathrm{p} 19 \mathrm{q}$ loss. Acta Neuropathol 126:267-276, 2013

4. Arita H, Narita Y, Yoshida A, Hashimoto N, Yoshimine T, Ichimura K: IDH1/2 mutation detection in gliomas. Brain Tumor Pathol 32:79-89, 2015

5. Balss J, Meyer J, Mueller W, Korshunov A, Hartmann C, von Deimling A: Analysis of the IDH1 codon 132 mutation in brain tumors. Acta Neuropathol 116:597-602, 2008

6. Casar-Borota O, Øystese KAB, Sundström M, Melchior L, Popovic V: A high-throughput analysis of the IDH1(R132H) protein expression in pituitary adenomas. Pituitary 19:407414, 2016

7. Dang L, White DW, Gross S, Bennett BD, Bittinger MA, Driggers EM, et al: Cancer-associated IDH1 mutations produce 2-hydroxyglutarate. Nature 462:739-744, 2009

8. Hao S, Hong CS, Feng J, Yang C, Chittiboina P, Zhang J, et al: Somatic IDH1 mutation in a pituitary adenoma of a patient with Maffucci syndrome. J Neurosurg 124:1562-1567, 2016

9. Hasegawa H, Shin M, Kondo K, Hanakita S, Mukasa A, Kin $\mathrm{T}$, et al: Role of endoscopic transnasal surgery for skull base chondrosarcoma: a retrospective analysis of 19 cases at a single institution. J Neurosurg 128:1438-1447, 2018

10. Hirata M, Sasaki M, Cairns RA, Inoue S, Puviindran V, Li WY, et al: Mutant IDH is sufficient to initiate enchondromatosis in mice. Proc Natl Acad Sci U S A 112:2829-2834, 2015

11. Horbinski C: What do we know about IDH1/2 mutations so far, and how do we use it? Acta Neuropathol 125:621-636, 2013

12. Ichimura K: Molecular pathogenesis of IDH mutations in gliomas. Brain Tumor Pathol 29:131-139, 2012

13. Ikota H, Nobusawa S, Tanaka Y, Yokoo H, Nakazato Y: High-throughput immunohistochemical profiling of primary brain tumors and non-neoplastic systemic organs with a specific antibody against the mutant isocitrate dehydrogenase 1 R132H protein. Brain Tumor Pathol 28:107-114, 2011 
14. Moriya K, Kaneko MK, Liu X, Hosaka M, Fujishima F, Sakuma J, et al: IDH2 and TP53 mutations are correlated with gliomagenesis in a patient with Maffucci syndrome. Cancer Sci 105:359-362, 2014

15. Mukasa A, Takayanagi S, Saito K, Shibahara J, Tabei Y, Furuya K, et al: Significance of IDH mutations varies with tumor histology, grade, and genetics in Japanese glioma patients. Cancer Sci 103:587-592, 2012

16. Pansuriya TC, van Eijk R, d'Adamo P, van Ruler MAJH, Kuijjer ML, Oosting J, et al: Somatic mosaic IDH1 and IDH2 mutations are associated with enchondroma and spindle cell hemangioma in Ollier disease and Maffucci syndrome. Nat Genet 43:1256-1261, 2011

17. Parsons DW, Jones S, Zhang X, Lin JCH, Leary RJ, Angenendt $\mathrm{P}$, et al: An integrated genomic analysis of human glioblastoma multiforme. Science 321:1807-1812, 2008

18. Pearce P, Robertson T, Ortiz-Gomez JD, Rajah T, Tollesson G: Multifocal supratentorial diffuse glioma in a young patient with Ollier disease. J Clin Neurosci 19:477-478, 2012

19. Ranger A, Szymczak A: Do intracranial neoplasms differ in Ollier disease and maffucci syndrome? An in-depth analysis of the literature. Neurosurgery 65:1106-1115, 2009

20. Shin M, Kondo K, Hanakita S, Hasegawa H, Yoshino M, Teranishi Y, et al: Endoscopic transsphenoidal anterior petrosal approach for locally aggressive tumors involving the internal auditory canal, jugular fossa, and cavernous sinus. J Neurosurg 126:212-221, 2017

21. Suzuki H, Aoki K, Chiba K, Sato Y, Shiozawa Y, Shiraishi Y, et al: Mutational landscape and clonal architecture in grade II and III gliomas. Nat Genet 47:458-468, 2015

22. Ueki K, Nishikawa R, Nakazato Y, Hirose T, Hirato J, Funada N, et al: Correlation of histology and molecular genetic analysis of 1p, 19q, 10q, TP53, EGFR, CDK4, and CDKN2A in 91 astrocytic and oligodendroglial tumors. Clin Cancer Res 8:196-201, 2002
23. Yan H, Parsons DW, Jin G, McLendon R, Rasheed BA, Yuan W, et al: IDH1 and IDH2 mutations in gliomas. N Engl J Med 360:765-773, 2009

24. Yang H, Ye D, Guan KL, Xiong Y: IDH1 and IDH2 mutations in tumorigenesis: mechanistic insights and clinical perspectives. Clin Cancer Res 18:5562-5571, 2012

\section{Disclosures}

The authors report no conflict of interest concerning the materials or methods used in this study or the findings specified in this paper.

\section{Author Contributions}

Conception and design: Tanaka, Nejo, Mukasa. Acquisition of data: Tanaka, Nejo, Ikemura, Nomura, Shin, Ushiku, Shibahara, Mukasa. Analysis and interpretation of data: Tanaka, Nejo, Ikemura, Nomura, Ushiku, Shibahara, Mukasa. Drafting the article: Nejo. Critically revising the article: Tanaka, Ikemura, Nomura, Takayanagi, Shin, Ushiku, Shibahara, Saito, Mukasa. Reviewed submitted version of manuscript: all authors. Approved the final version of the manuscript on behalf of all authors: Tanaka. Study supervision: Tanaka, Mukasa.

\section{Correspondence}

Shota Tanaka: Graduate School of Medicine, The University of Tokyo, Japan.tanakas-tky@umin.ac.jp. 